\title{
Study on SOC Estimation Based on Circular Optimization for RBF Neural Network
}

\author{
Tiezhou Wu, Xiaomin Wu, Mengmeng Yang and Meng Luo \\ Hubei Collaborative Innovation Center for High-efficiency Utilization of Solar \\ Energy \\ Hubei University of technology, Wuhan 430068, China \\ wtz315@163.com,404675421@qq.com, \\ 982173002@qq.com,392249972@qq.com
}

\begin{abstract}
This paper proposed a circular particle swarm optimization least squares (CPSOLS) method which is consisted of the regularized least squares $(R L S)$ method and the adaptive particle swarm optimization (APSO) algorithm. The RLS algorithm optimized the parameters of the RBF network, aiming at the phenomenon of RLS trapping in the local minimum, introduced the penalty factor and used the global optimization ability of the particle swarm optimization algorithm to make it out of the local minimum; simplified the structure of the RBF network and improved the generalization ability of the network. The APSO algorithm weakened the precocious converge phenomena of the particle swarm optimization algorithm, adopted the adaptive selection of the nonlinear dynamic inertia weight which is guided by the control factor of the battery external characteristic temperature parameters, optimized the link weight of the RBF network, improved the state of charge (SOC) estimation accuracy and real-time performance of the RBF network. Using the Arbin multifunctional battery test system BT2000 to collect the sample data of the battery external characteristic parameters, and using the sample data to train and optimize the RBF neural network, and estimate the SOC of the batteries. The results showed that the optimized RBF network improved the SOC estimation accuracy and real-time performance.
\end{abstract}

Keywords: Regularized least squares, Adaptive particle swarm optimization algorithm, RBF network, Control factor, SOC estimation

\section{Introduction}

State of charge (SOC) is one of the key parameters of power battery energy management [1]. Accurate estimation of the SOC cannot only respond the batteries charging and discharging status, protect and extend the batteries' life, but also can timely adjust the charging and discharging control strategy according to the SOC [2].

Comparison of radical basis function (RBF) neural network and artificial neural network (ANN) and genetic algorithm (GA) neural network is simple structure, short training time, and with the ability to approximate any nonlinear function [3]. The RBF neural network has higher complexity in the process of building networks and selecting the network parameters, in order to weaken complexity of selecting network and simplify the constructed network [4]. In this paper chooses the regular least squares (RLS) to build the RBF neural network. The RLS algorithm selects a suitable center value for the RBF neural network from the sample to avoid excessive interference in the sample affect the generalization ability of the system [5]. To eliminate the RLS trapping in local minimum conditions and affect the generalization ability of the system, introduced adaptive particle swarm optimization (APSO) algorithm for global optimization. APSO is widely applied to 
optimize the neural network system, due to its advantage of simple principle, easy to implement, a small amount of calculation and high efficiency, and the APSO improved the PSO algorithm in the terms of easy to trap in local minimum and inertia weight effect the global search capability [6].

The APSO and RLS algorithm were combined in this paper, and a circular particle swarm optimization least squares (CPSOLS) method was proposed to train the RBF neural network and obtain a set of weights, centers and widths of the RBF neural network, and to estimate the SOC of lithium-ion batteries [7-8]. The batteries external characteristic parameters as the input sample space of the RBF neural network could enhance the selected fitness of the RBF neural network parameters. When adjusted the link weights of the RBF network, the introduction of the temperature parameter control factor in the battery charging and discharging process as the guide improved the estimation accuracy and the real-time performance for the RBF neural network estimating the SOC of lithium-ion batteries.

\section{Circular Optimization RBF Neural Network Algorithm}

The RBF neural network is a forward network model with simple structure, good approximate the nonlinear function and learn by itself. And now it is widely used in the field of function approximation, prediction and pattern recognition etc. This section, the APSO and RLS algorithm was combined with each other to optimize the structure and parameters of the RBF network, and improved the calculation accuracy and the convergence rate of the RBF neural network [9-10].

\subsection{Principle of RLS Algorithm}

The RLS algorithm is a linear regression algorithm with high speed and efficiency that can improve the generalization ability of the RBF neural network. According to the linear regression model, RBF neural network is a 3-layer network, adopted the Gaussian function as the base function of the RBF network [11]. So the desired output of the network, using the following equation:

$$
Y=G_{r}(x)+e=\sum_{i=1}^{n} \theta_{i} \exp \left(\frac{-\left\|x-c_{i}\right\|^{2}}{\rho}\right)+e
$$

where ${ }^{x}$ is the input vector of the network, ${ }^{\theta_{i}}$ and ${ }^{c_{i}}$ are the link weight and node center of hidden layer of the i-th node in the hidden layer, ${ }^{\rho}$ is the variance of the network-based functions, $e$ is the output error.

Supposed that there are $\mathrm{N}$ samples, $\{y(i), x(i)\}_{i}^{n}$ is a sample space of the network. $x(i)$ is the input sample of the network, ${ }^{y(i)}$ is the corresponding desired output of the input sample. Set the center values of the basic function are selected from the samples, each sample views as a center value of the basic functions individually. Then $c_{i}=x(i)(1<i<n)$, set the variance of the basic functions is $\rho$, gives: $\delta(i)=\operatorname{ex}\left(\frac{-\| x\left(i+c_{i} \|^{2}\right.}{\rho}\right)$

Then the desired output of the network is as follows:

$$
Y(i)=\varphi \delta(i)+e(i)=\sum_{1}^{n} \theta_{i} \delta(i)+e(i)(1<i<n)
$$

where ${ }^{e(i)}$ is the errors between the actual output ${ }^{\varphi(i)}$ and the desired outputs ${ }^{Y(i)}$.

Using the Gram-Schmidt method to decompose the regression function ${ }^{\varphi}$, 
then ${ }^{\varphi}=Q A$, where $Q$ is an orthogonal matrix, satisfied $Q_{i}{ }^{T} Q_{j}=1(i \neq j)$. A is an $\mathrm{NxN}$ upper triangular matrix, the main diagonal are all 1.

The desired output of network Eq. (3) can be changed as:

$$
\left.Y(i)=\emptyset A^{-1}\right)\left(A \Phi \quad \rightarrow e_{i} \quad \Rightarrow Q g+i t\right.
$$

where $g=A \Phi, \Phi=\left[\theta_{1}, \theta_{2}, \ldots \theta_{n}\right]^{T}$ is the links weight parameter vector.

Regularization method added an error term in the regression model, so that the model will not be too complicated. General regularization error can be defined as:

$E(F)=\frac{1}{2} \sum_{i=1}^{n}\left(y_{i}-F\left(x_{i}\right)\right)^{2}+\lambda E_{R}(F)$

where ${ }^{\frac{1}{2} \sum_{i=1}^{n}\left(y_{i}-F\left(x_{i}\right)\right)^{2}}$ is the standard error; $F$ is the approximation function, $\lambda$ is the regularization coefficient, $0<\lambda<1 ;{ }^{E_{*}(F)}$ is the regularization term.

Therefore, the criterion error function which used in the RLS algorithm is: ${ }^{J_{R}}(\mathrm{~g}, \lambda)=e^{T} e+\lambda g^{T} g, \lambda g^{T} g$ is the regularization term. According to $\partial J / \partial g=0$, so the error under the regular standardization will be defined as $[\text { err }]_{i}=\left(Q_{i}^{T} Q_{j}+\lambda\right) g_{i}{ }^{2} / Y^{T} Y$

When the RLS algorithm selected regression factors, a given tolerance error is defined as: ${ }^{1-} \sum_{i=1}^{n_{r}}[\mathrm{err}]_{i}<\xi$, where $0<\xi<1, n_{r}$ is the number of the final regression factors.

In the process of selecting the hidden layer node center of the RBF network, in order to avoid the algorithm trapped in a local minimum, sometimes may need to know the total error between the current network and all samples during the operation, so evaluation of the total error of the current network is given by

$$
\text { err }=\sum_{s 1}^{m} \sum_{s 2}^{o}(\bar{Y}-Y)
$$

where ${ }^{m}$ and $\mathrm{o}$ are the number of the hidden layer unit and output layer unit, respectively.

$Y$ is the actual output value of the network, $Y$ is the desired outputs value.

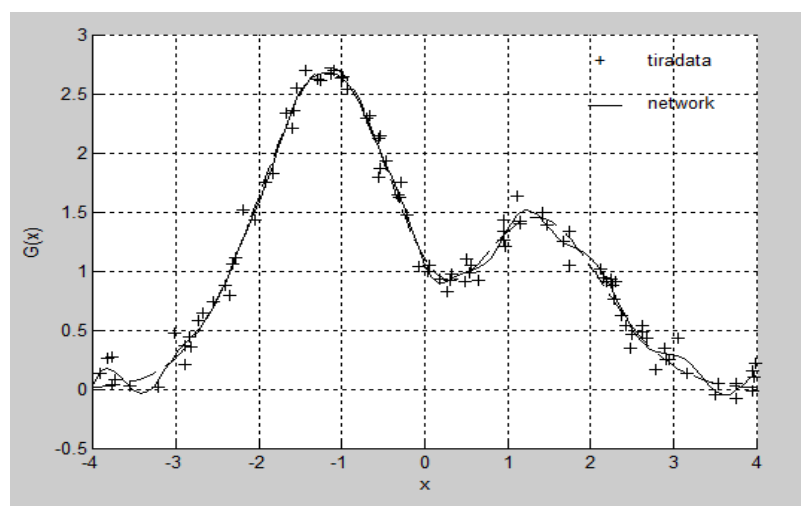

Figure 1. The Approximation Curve of RLS Optimization Selected the Network Parameters 
When RLS algorithm selected the hidden layer node center, the evaluation error resulted by the RBF network will oscillate, most of them recover after oscillating in a few times and the network had been trapped in a local minimum. To avoid this situation, introduced a penalty factor $\mathrm{H}$ in the algorithm. The penalty factor will make the RLS algorithm come back to the step before trapping in the local minimum and continuous oscillate $\mathrm{H}+1$ times , and will judge this time whether the algorithm has been out of the local minimum after oscillating stable. The approximation curve of the RLS optimization selected the network parameters as shown in Figure 1.

Introduced penalty factor conditions not only make the RLS algorithm avoid trapping in a local minimum, but also avoid further operating after the network reaching the optimal network structure, and improve the real-time performance of the algorithm.

\subsection{Adaptive Particle Swarm Optimization}

PSO is a new global optimization algorithm between group organizations and individuals with simple optimization strategy, fast convergence, stability and can be distributed to achieve. The search space of PSO algorithm is constituted by a number of "particles". The particles which affect by their velocity ${ }^{v_{i}}$, their location $x_{i}$ and their optimal history position information of their groups, can quickly find the global optimum solution in the search space [12]. But the PSO algorithm in the search process will plunge into local optimum, that will result in the so-called precocious converge phenomenon, which will affect the optimization search ability of the algorithm. To avoid precocious converge phenomenon, generally adjust the inertia weight of the PSO algorithm, and enhance the search ability of the algorithm in global and local [13].

When selected the inertia weight, if the inertia weight is larger, the global search ability of the algorithm is strong, but the local search ability is weak; although fast convergence, but not easy to get an accurate solution. When the inertia weight is smaller, the local search ability of the algorithm is strong, the global search ability is weak, this is favorable to the local search, but the convergence of the algorithm is slow and may trap in the local minima. The update formula of the space particle velocity and location as follows:

$v_{i d}=\omega v_{i d}+c_{1} r_{1} \times\left(p_{i d}-x_{i d}\right)+c_{2} r_{2} \times\left(g_{i d}-x_{i d}\right)$

$x_{i d}=x_{i d}+v_{i d}$

Common linear inertia weight reduction strategy, the convergence of the PSO algorithm is slow later and need to predict the maximum number of iterations, the selected inertia weight can't better meet the requirements of the global search and local search. Thus proposed an adaptive coefficient instead of the liner reduction optimization strategy, referenced the variation idea, increased the diversity of the particles, and enhanced the ability of the global search and local search of the algorithm.

In order to better balance the contradictory between the global search and the local search, enhance the ability to deal with nonlinear problems, introduced a nonlinear adaptive inertia weight, the update equation of the weight is as follows: $\omega=\omega_{e}-\left(\omega_{s}-\omega_{e}\right) \exp \left[-k *\left(\frac{t}{t_{\max }}\right)^{2}\right]$

where $\omega$ is the actual inertia weight, $\omega_{s}=0.9, \omega_{e}=0.4 .{ }^{t}$ is the iteration number, and introduced the control factor $k$ to adjust the fitness of the change curve between $\omega$ and $t$.

$$
k=\int_{t_{0}}^{t_{n}} u(t)
$$


where ${ }^{t_{0}},{ }^{t_{n}}$ is the collecting temperature moments, ${ }^{u(t)}$ is the function of temperature parameters.

The selection of the control factor $k$ depends on the characterization of the battery temperature parameters [14]. Battery capacity changes with the temperature changes, it is necessary to adjust the inertia weight based on the reasonable variations of the actual temperature. In the low-temperature phase, as the temperature drops, its discharge capacity is gradually declining. Temperature drops speed, the declining rate of capacity has accelerated. In the high-temperature phase, its capacity did not change significantly, and the maximum capacity of the reference did not change significantly, but if the temperature is too high, the capacity curve falling instead of rising.

In the process of the battery charging and discharging, the battery temperature is constantly changing, fully considering the influence of temperature brought to the battery can more accurately estimate the battery SOC.

\subsection{Using CPSOLS Optimize RBF}

2.3.1 Principle of CPSOLS: To achieve the structure optimization of the RBF network, combined APSO and RLS, and made the APSO optimized parameters process and the RLS algorithm together, the unknown parameters $(\lambda, \rho)$ of the RLS algorithm and the hidden layer node center of the RBF network, the number of the nodes and the links weight are optimization selected at the same time.

Table 1. RLS Algorithm Selected the Date Samples of H Values

\begin{tabular}{cccccc}
\hline \hline No. & Current $(A)$ & Voltage $(V)$ & Temperature $\left.^{\circ} \mathrm{C}\right)$ & Charge_Capacity $($ Ah) & Charge_Energy $($ Wh $)$ \\
\hline 1 & 0.977639973 & 0.991987705 & 15.13 & 0.742151081 & 2.925004554 \\
2 & 0.98623991 & 0.992604077 & 15.57 & 0.742698729 & 2.925547914 \\
3 & 0.977639973 & 0.992295861 & 15.83 & 0.745988033 & 2.928812167 \\
4 & 1.983831406 & 1.999791026 & 18.25 & 0.749710446 & 2.933363778 \\
5 & 1.983831406 & 2.000099182 & 18.73 & 0.751928323 & 2.93779936 \\
6 & 1.983831406 & 2.000099182 & 19.05 & 0.761908712 & 2.957759892 \\
7 & 2.981422901 & 3.005128622 & 21.32 & 0.763222697 & 2.961001864 \\
8 & 2.972822905 & 3.005436897 & 21.67 & 0.764885516 & 2.965999226 \\
9 & 2.972822905 & 3.005745173 & 21.92 & 0.783175257 & 3.020968411 \\
10 & 3.979014397 & 4.012931824 & 23.42 & 0.792054363 & 3.056541381 \\
11 & 3.979014397 & 4.012931824 & 23.87 & 0.807623826 & 3.119020903 \\
12 & 3.979014397 & 4.013240337 & 24.12 & 0.809847998 & 3.127946811 \\
13 & 4.976605892 & 5.028440475 & 26.27 & 0.819288757 & 3.174430581 \\
14 & 4.976605892 & 5.028131962 & 26.75 & 0.824852488 & 3.20240693 \\
15 & 4.98520565 & 5.028131962 & 27.08 & 0.83876182 & 3.272345725 \\
\hline \hline
\end{tabular}

When selecting the value of the penalty factor $\mathrm{H}$, let formula (7) of the evaluation error function as the core to establish a test example for an oscillating curve. Some parameters in the process of the lithium-ion batteries charging and discharging as the data samples of the example are listed in Table 1 to test and select the value of $\mathrm{H}$. When the evaluation error trapping in oscillating, the local in minimum. To make it jump out of the local minimum quickly, activated the penalty factor in the evaluation error, the oscillating range of the evaluation was expanded, the oscillation of the evaluation error recovered, and the RLS algorithm jumped out of the local minima. Figure 2, illustrated that the oscillation of the test evaluation error quickly recover after the penalty factor $\mathrm{H}$ working. After continuous oscillation 5 times, the curve started to fall and then recover. After several instances of the test, generally when the value of $\mathrm{H}$ selects 5 , the range of the evaluation error reached its peak, and then began to decrease. 


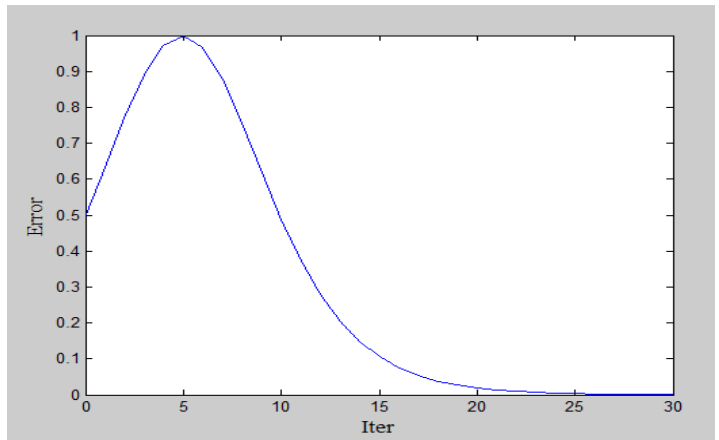

Figure 2. Oscillation Curve of the Evaluation Error

After the RLS algorithm optimized the parameters of the RBF network, APSO algorithm searched a series of solution space that has been optimized by the RLS. Under the nonlinear dynamic weight adjustment strategy which is guided by the control factor ${ }^{k}$, APSO algorithm can better adjust the unbalanced phenomenon of the global search and local search which occurred in the process of the global optimization, and weaken the problem that in the late search into the local optimization minimum. APSO global optimization algorithm can find the optimal solution from the solution space of the all possible combinations of the unknown parameters in the RLS algorithm to optimize the structure of the RBF network.

In the process of the CPSOLS optimization algorithm, the selection of the RBF parameters, link weights and $(\lambda, \rho)$ of the RLS algorithm, and the inertia weight of the APSO algorithm search space are adjusted and optimized continuously. That makes the structure of the RBF network be simplify, operation speed and helps to improve the calculation accuracy.

2.3.2. RBF Network Optimization: Adopting the CPSOLS method to global optimize the parameters of the RBF network and the regularization coefficient of the RLS algorithm. In the parameter optimization, the search interval of the regularization coefficient ${ }^{\lambda}$ of the RLS algorithm is $[0,1]$, the search interval of the variance of the network function ${ }^{\rho}$ is $[0.0001,1]$, the particles swarm number of the APSO algorithm is $\mathrm{N}$ $=50$. The CPSOLS optimize RBF network method is described in the following steps:

Step1): Create the sample data. The sample data includes terminal voltage, discharge current, temperature.

Step2): Normalize all of sample data.

Step3): Use RLS algorithm to optimal select the parameters of the RBF network (link weights, centers and widths).

Step4): Judge this time whether the algorithm has trapped in the local minimum or not. If it has trapped in the local optimization minimum, then the penalty factor starts to oscillate $\mathrm{H}+1$ times continuously.

Step5): Judge whether the evaluation error function has recovered and has been out of the local optimization minimum or not.

Step6): The optimized parameters of the RLS algorithm compose the search space of the APSO algorithm, and encode the parameters.

Step7): Update the particle velocity, location and inertia weight.

Step8): Random search again in the search space. If the fitness of the searched new location is better than the current location, then the new location as a starting point for the next iteration. Or, the previous location as the starting point for the next evolution.

Step9): Judge whether satisfy with the termination condition of the APSO algorithm or 
not. If satisfied, the APSO algorithm stops and steers to next step, and then export the function parameters of the RBF neural network (center value and width). Or, steers to Step 3, and continue iteration.

Step10): Construct the RBF neural network based on the function parameters obtained from the Step 9. And obtain the link weights of the RBF network by calculating.

Step11): Judge whether satisfy with the termination condition of the RBF algorithm or not. If satisfied, the algorithm ends, and the CPSOLS-RBF neural network learning and training ands, and obtain the completed training neural network. Or, steers to Step 3, and continue iteration.

\section{SOC Estimation Experimental Verification}

Define the external characteristics parameters of the battery as the input vector of the RBF network input layer, the number of nodes in the hidden layer is 33 optimized by the CPSOLS algorithm. The RBF network output layer is the battery estimated SOC. The block diagram of the algorithm of the SOC estimation is shown in Figure 3.

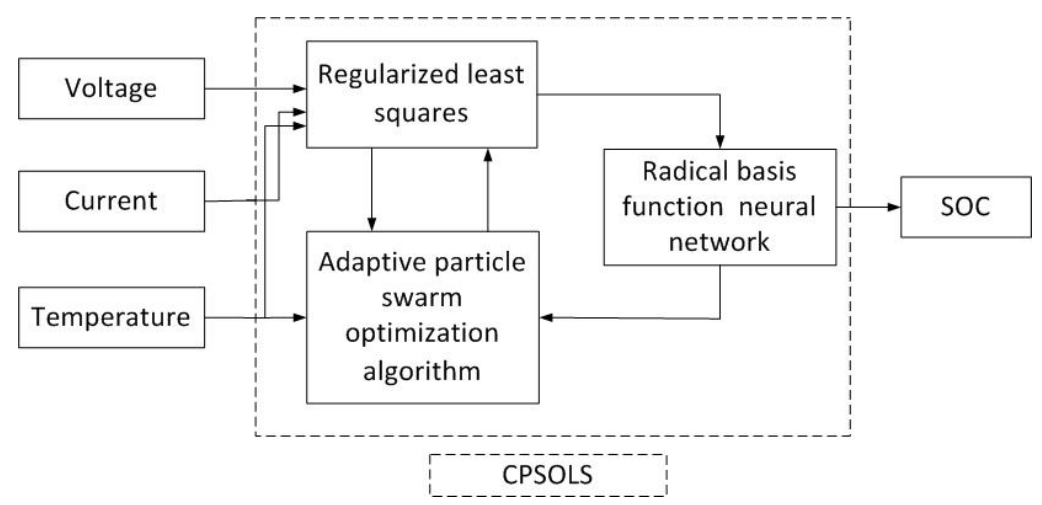

Figure 3. The Block Diagram of the RBF Network Estimated the SOC

The experiment data used for this study can be obtained from the Arbin multifunctional battery test system BT2000 made by US. The voltage of the testing battery is $7.4 \mathrm{~V}$, the capacity of the combined battery is 5.2AH. The single lithium-ion batteries are produced by Panasonic, the CGR18650CH module, with a nominal voltage of $3.6 \mathrm{~V}$ and a nominal capacity of $2500 \mathrm{mAh}$. The battery connected mode is two series and parallel connection.

To verify the efficiency of the method, respectively adopted the GA and CPSOLS-RBF method to estimate SOC, and compared the estimation result of the two methods. The collected sample data as a set of input samples of the two methods.

In the data collection process, respectively adopted different charge and discharge current and recorded the battery external characteristic parameters (terminal voltage, the discharge current and temperature). The different discharge current is respectively divided into 10 different intervals, there are 50 simple data in every interval. The entire discharge process consists of 1500 samples.

Through different current battery charge and discharge test, set up the Arbin battery test system BT2000 to record once data every two seconds, and recorded the battery parameters. Figure 4, shows the charge-discharge current curve and the terminal voltage under the different currents conditions, the voltage curve. Figure 5, presents the temperature changing curve in the test process. 


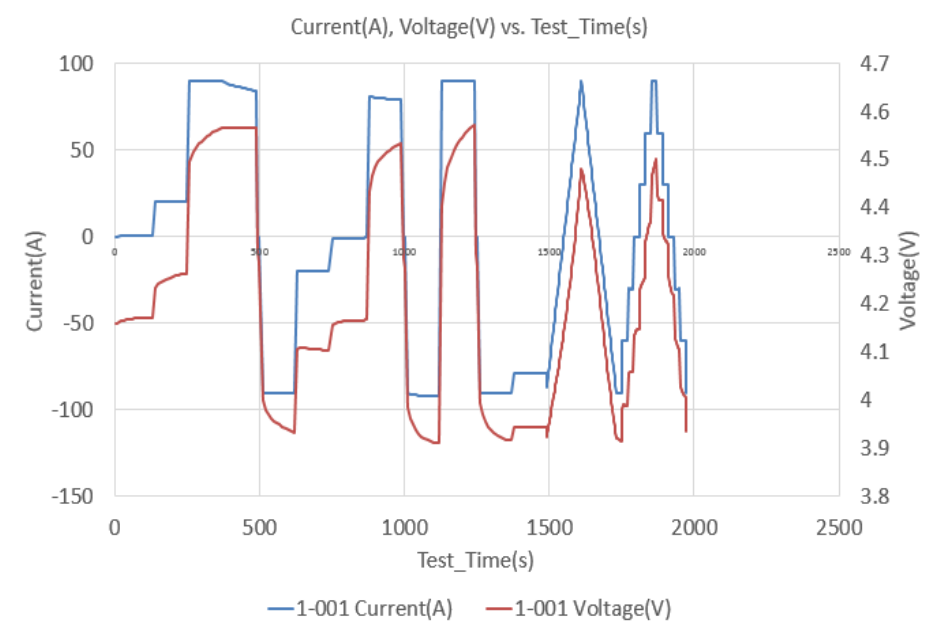

Figure 4. The Charge-Discharge Current Curve and the Terminal Voltage Under the Different Currents

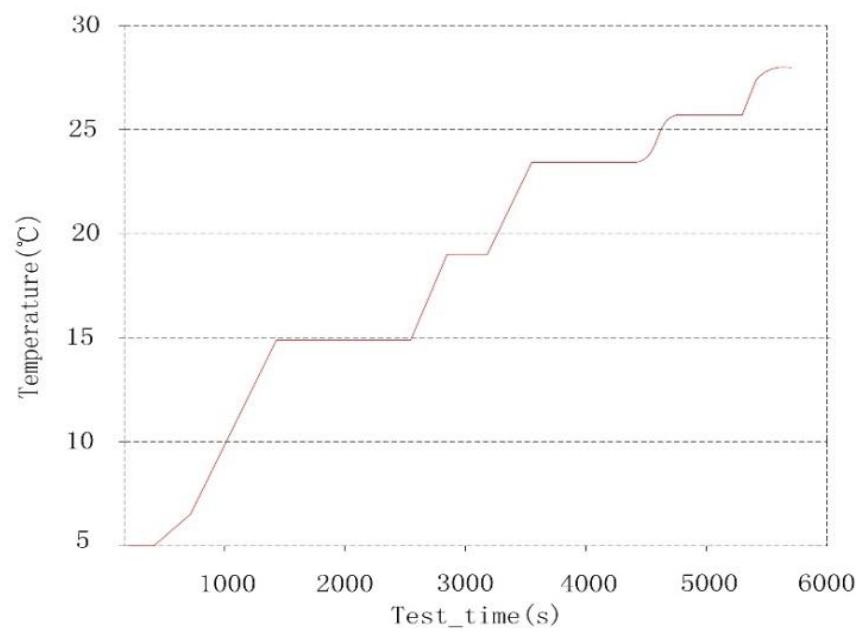

Figure 5. The Temperature Changing Curve in the Charging and Discharging

\section{Process}

Use the collected sample data as the input vector of the two algorithms for the data simulation experiments, respectively. The same sample data respectively input to the GA network and CPSOLS-RBF network, the fitness of the CPSOLS-RBF network is improved, and the particles search speed in the search space is better than the GA network which is not optimized. From Figure 6, it can be see that the adaptive dynamic inertia weight of the circular optimization algorithm makes the particles search ability to strengthen, speeds up the global and the local search ability. 


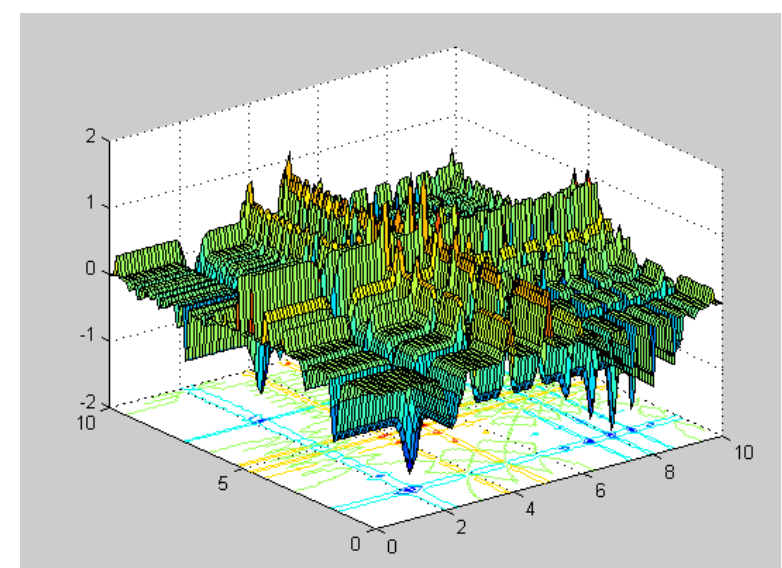

\section{Figure 6. The Adaptive Inertia Weight Strategy Speeds up the Particles Search}

Through the training for the data samples under different discharge currents, compared the simulation results of the above-mentioned two methods which are estimated SOC, it can be seen that the training speed and the estimation accuracy of the CPSOLS-RBF network is better than the general GA network.

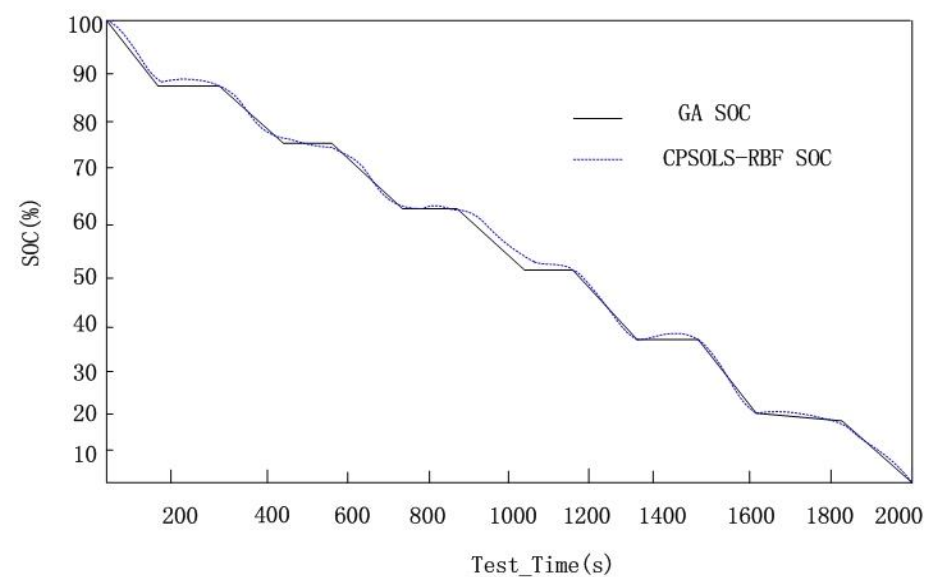

(a)

Figure 7. Comparison of the SOC Estimation Results

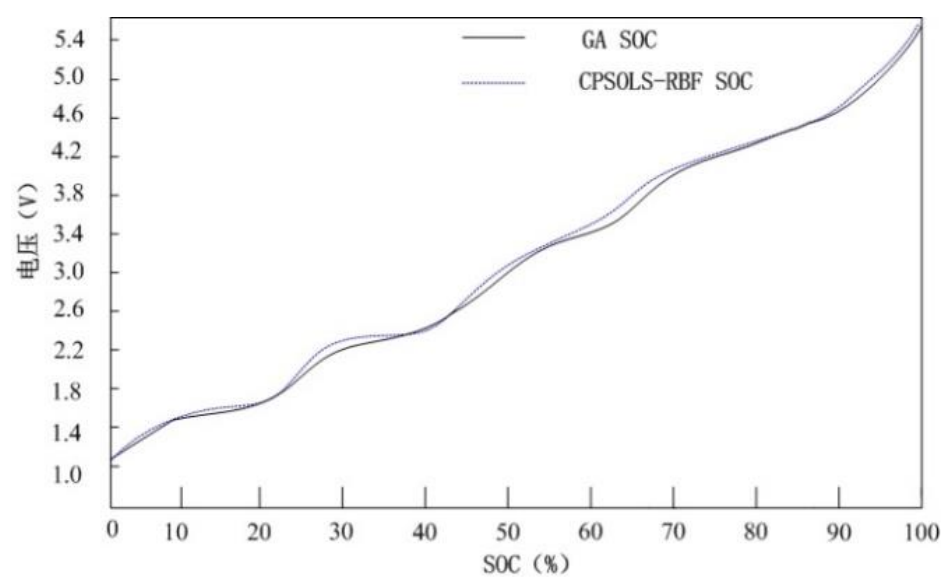

(b)

Figure 7. Comparison of the SOC Estimation Results 
A comparison of the estimation result for these two methods is shown in Figure 7: the output accuracy of the optimized RBF network is obviously higher than the normal GA network. From Figure 7, it can be seen that the algorithm which is optimized by the CPSOLS method improved the generalization ability of the RBF network. Used it to estimate the lithium-ion batteries SOC, the results in the Figures showed that the SOC estimation accuracy of the optimized RBF network is improved significantly.

\section{Conclusion}

This paper combined the RLS method and the APSO algorithm, proposed a circular particle swarm optimization least squares (CPSOLS) method to optimize the structure and parameters of the RBF network. The RLS algorithm optimized the parameters of the RBF network, and used the APSO algorithm to optimize its own regularization coefficient and weaken the phenomenon of trapping in the local optima. The APSO algorithm adopted the nonlinear dynamic adaptive adjustment strategy which is guided by the temperature external characteristic parameters of the lithium-ion battery to select the inertia weights. The adaptive adjustment for the inertia weight is help to strengthen the global and local search ability of the PSO algorithm, and speed up the processing rate of the algorithm. After optimization of the CPSOLS method, the structure of the RBF neural network became more simplified, and the generalization ability was improved. With the continuously training for the sample data which is consisted by the lithium-ion battery parameters (terminal voltage, the discharge current and temperature), the algorithm is faster and more proficient for the operation of the data, and the estimation accuracy of the battery SOC is also continuously improved in the optimization process.

\section{Acknowledgment}

This work was supported by Science and Technology Department of Hubei province of china science and technology research key project (No. 2010CDB05802). And Research Fund for the Doctoral Program of Hubei University of Technology (No. BSQD12018).

\section{References}

[1] Z. Yun, Z. Cheng-Hui, C.Na-Xin. "Estimation of state-of-charge of lithium-ion battery: Nonlinear observer method $[\mathrm{J}]$ ". Control Theory and Applications, 12, 29(2012).

[2] Vogelsang Timothy J, Wagner Martin. Integrated modified OLS estimation and fixed-b inference for cointegrating regressions $[\mathrm{J}]$. Journal of Econometrics. $\mathrm{v} 2$ 2, 178(2014).

[3] M. Bijanzadeh, D. Kahani, E. Kohan Daneshi, M. Joghatai, SC Gharooni, M O TokhiNARMAX-OLS Representation of a semi-active dynamic leg joint model for a paraplegic subject using functional electrical stimulation [C]. Lecture Notes in Engineering and Computer Science,(2013)July3-5; London, United kingdom

[4] N. Kazuteru, L. Fabrizio, "A novel scheme for concurrent error detection of OLS parallel decoders [C]", IEEE International Symposium on Defect and Fault Tolerance in VLSI Systems, (2013)October2-4;New York City, NY, United states

[5] C. Wen-Yeau, "Estimation of the state of charge for a LFP battery using a hybrid method that combines a RBF neural network, an OLS algorithm and AGA [J]", International Journal of Electrical Power and Energy Systems.1, 53(2013).

[6] L. Jianwei, J. Bin, M. Michael, X. Ming, "On-line battery state of charge estimation using Gauss-Hermite quadrature filter [C]", IEEE Applied Power Electronics Conference and Exposition, (2012)February5-9; Orlando, FL, United states

[7] D. Dajun, L. xue, F. Minrui, I. George W. "A novel locally regularized automatic construction method for RBF neural models [J]”, Neurocomputing. 12, 98 (2012)

[8] H. Cui, W. Buying, L. Weiming. "Short-term forecasting of wind power output based on improved OLS-RBF ANN model [J]", Electric Power Automation Equipment.9, 32 (2012).

[9] A B Hashemi , M.R. Meybodi, "A note on the learning automata based algorithms for adaptive parameter selection in PSO [J]”, Applied Soft Computing Journal.11, 1(2011). 
[10] N. Peng, J. Geng, Q. Zhiguang, "Self-adaptive Inertia Weight PSO Test Case Generation Algorithm Considering Prematurity Restraining [J]", JDCTA: International Journal of Digital Content Technology and its Applications.5, 9 (2011).

[11] S. Masoud, E. Tarek Y, "Multi-objective optimal design of hybrid renewable energy systems using PSO-simulation based approach [J]", Renewable Energy. 8, 68 (2014)

[12] H. Wei, W. Nicholas, C. Chaochao, P. Michael. State of charge estimation for Li-ion batteries using neural network modeling and unscented Kalman filter-based error cancellation [J]. International Journal of Electrical Power \& Energy Systems. 11, 62(2014)

[13] C. Hao, G. Yu, H. Xia. "Adaptive modelling with tunable RBF network using multi-innovation RLS algorithm assisted by swarm intelligence [C]", IEEE International Conference on Acoustics, Speech and Signal Processing - Proceedings, (2011) May22-27; Prague, Czech republic

[14] X. Rui, S. Fengchun, G. Xianzhi, G. Chenchen, "A data-driven based adaptive state of charge estimator of lithium-ion polymer battery used in electric vehicles [J]", AppliedEnergy.1, 113(2014)

\section{Authors}

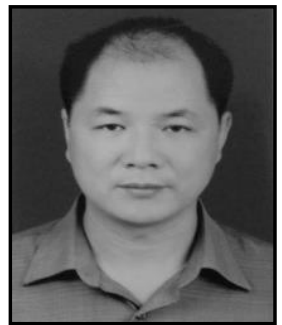

Tiezhou Wu was born in Hubei, China. He received the Master degree in Computer application technology from Naval University of Engineering and the Ph.D. degree in system analysis and integration from Huazhong University of Science and Technology. $\mathrm{He}$ is currently a Professor of Institute of electrical and electronic engineering, Hubei University of Technology. His research interests include photovoltaic power generation, energy storage technology, and system analysis and integration.

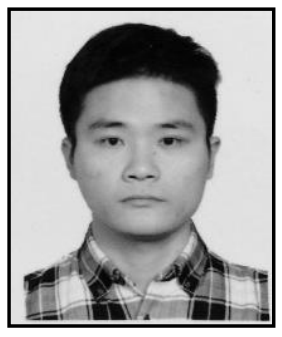

Xiaomin Wu was born in Jiangxi, China. He is currently a student of Institute of electrical and electronic engineering, Hubei University of Technology. He research interests include photovoltaic power generation and energy storage technology.

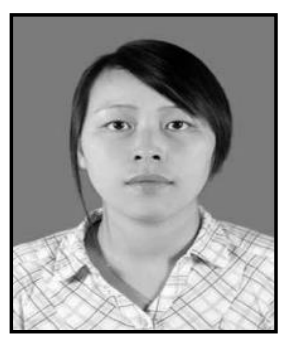

Mengmeng Yang was born in Hubei, China. She is currently a student of Institute of electrical and electronic engineering, Hubei University of Technology. Her research interests include photovoltaic power generation and battery fast charging.

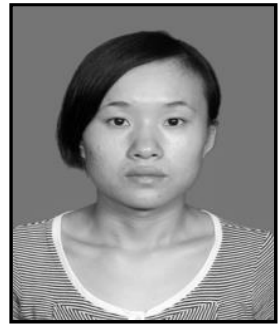

Meng Luo was born in Hubei, China, 1991. She is currently with the M.Sc. degree of Electrical Engineering, Hubei University of Technology, China, as a student. Her research interests is energy storage technology. 
International Journal of Grid Distribution Computing

Vol. 8, No.6, (2015) 